\title{
EL IMPACTO DE LA INMIGRACIÓN EN NAVARRA: FUSTIÑANA, UN CASO PARTICULAR
}

\author{
The impact ot the inmigration in Navarra: Fustiñana, \\ a particular case
}

\author{
Alberto Fernández Puig \\ Recibido 03/03/2007; aceptado el 20/06/2007
}

Resumen. El presente trabajo tiene por objeto el estudio de la evolución de la inmigración en Fustiñana. Este fenómeno presenta ciertas peculiaridades dentro del contexto general de la inmigración en Navarra. Una de las principales características que aislan a Fustiñana del resto de la Comunidad Foral de Navarra es la casi total ausencia del Sector Secundario, lo cual hace de la presencia de inmigrantes en la localidad un hecho excepcional dentro del panorama general que la inmigración presenta en el resto de Navarra. Nuestro estudio, debido a su carácter local, puede ser extrapolado además a otras poblaciones limítrofes que, al igual que Fustiñana, poseen una fuerte presencia de población inmigrante sin tener tampoco un sector secundario desarrollado.

Palabras clave: Navarra, Fustiñana, inmigración..

Abstract. This research has the aim of studying the evolution of immigration in Fustiñana. This fact shows some peculiarities within the general context on immigration in Navarra. One of the main characteristics that emphasize Fustiñana from the rest of the community of Navarra is the quite total lack of secondary sector, so that the presence of immigrants in this place is exceptional comparing it with the general situation of immigration in the rest of Navarra. Our research, due to its local character, can be transferred to other communities that are near Fustiñana, just because in the same way, they have a strong presence of immigrants too, without having a developed secondary sector.

Key Words: Navarra, Fustiñana, inmigration.

\section{INTRODUCCIÓN:}

Fustiñana es una pequeña localidad situada en la Ribera de Navarra, bañada por el río Ebro, su economía tradicional ha sido hasta ahora la agrícola. A caballo entre la Vega del Ebro, lugar de extraordinaria fertilidad gracias al regadío, y la Bardena, calificado como desierto debido a su árido clima, sus gentes se han dedicado desde siempre a la agricultura intensiva en las zonas regables, donde se practica una agricultura a base de hortalizas, maiz, y alfalfa, y otra agricultura de carácter extensivo en el secano, donde se cultiva cereal por el sistema año vez.
Si la agricultura había sido la ocupación principal de los fustiñanenses desde antaño, sin embargo, la mecanización de las labores agrícolas, la pérdida de poder adquisitivo de los agricultores, los bajos salarios pagados a los peones $y$, sobre todo, la oferta de trabajo de las zonas de industrialización en las tradicionales áreas industriales del norte de España, empujaron, desde comienzos de los años sesenta a gran parte se sus habitantes a emigrar.

Este éxodo fue continuo y permanente hasta la Crisis del 73. La mayoria de los inmigrantes fustiñaneros optaron por acudir a la capital de Navarra, siendo de este modo Fustiñana, al igual que las localidades colindantes, el verdadero vi- 
vero que provocó el crecimiento de Pamplona. No sólo acudieron los emigrantes hacia esta ciudad, localidades como Bilbao, Barcelona y Zaragoza también acogieron a los emigrados de Fustiñana. El éxodo de los fustiñanenses se detuvo en parte gracias a la industrialización de la vecina ciudad de Tudela. Los habitantes de Fustiñana se desplazaban a diario a esta localidad, este hecho provocó que comenzase a tener importancia el sector secundario entre los trabajadores de este pueblo. La emigración de Fustiñana quedó detenida a mediados de los años setenta, continuando su población trabajando en las labores agrícolas. No descubrimos nada nuevo diciendo que la agricultura constituye un sector «refugion en tiempos de crisis. Decenas de albañiles en paro, parados de la industria, jóvenes que no se podian incorporar a un mercado laboral en recesión y mujeres que no tenían otra salida, dedicaban sus esfuerzos a la recolección del espárrago, tomate y cebolla

A mediados de los ochenta la economía se reactivó. En esta época tuvo lugar el último proceso de emigración en Fustiñana, decenas de jóvenes se marcharon a trabajar a Pamplona debido al fuerte desarrollo económico que existía en esta ciudad, sobre todo por la necesidad de trabajadores en la industria del automóvil y por la gran exigencia de nuevos funcionarios que demandaba la nueva Administración de una Autonomía recién estrenada.

El gran acontecimiento en cuanto a las migraciones se produce en Fustiñana a mediados de los años 90. Si hasta entonces había sido una localidad que emitía población a los centros económicos regionales más importantes, a partir de esta fecha comenzó a ser receptora de inmigrantes.

Estos primeros inmigrantes eran en su mayoría de nacionalidad búlgara, tras ellos se situaron ecuatorianos y marroquíes, a los cuales les acompañan personas de otras 15 nacionalidades diferentes, lo que al principio se vio como un hecho anecdótico y, en cierta manera exótico, pasó, en unos pocos años, a ser norma.
Con estos datos nos situamos en una situación excepcional, Fustiñana, localidad donde hasta los años noventa se había asistido a un imparable proceso emigratorio, comenzó a recibir población de lugares lejanos. Esta población se instala en la localidad de manera permanente y en la actualidad este aporte migratorio continúa. La presencia de inmigrantes a fecha de 31-7-2006 era de 236 personas, lo cual supone el 9'34\% del total de la población del municipio, valores que están muy por encima del 7'57\% de media en la Comunidad Foral de Navarra.

Con este trabajo pretendemos analizar la inmigración procedente del extranjero, su composición por nacionalidades, su incidencia en el empleo y en la vivienda, así como su importancia en el crecimiento global de la población en esta localidad.

\section{FUSTIÑANA: POBLACIÓN E INMIGRACIÓN.}

Si analizamos las series históricas sobre la población total de Fustiñana en los últimos veinte años comprobaremos como, hasta el año 2000, el crecimiento total de sus habitantes apenas si aumentaba, aún es más, existian años de receso demográfico, en concreto el año 1999 la cifra de población era de 2.290 habitantes, la más baja desde 1930 . Los datos hasta el año 2000 reflejan un crecimiento prácticamente nulo, cuando no una pérdida de población, de la Villa de Fustiñana. Estas variaciones residenciales de Fustiñana están relacionadas con el fenómeno de la emigración de los noventa descrito más arriba y con el nulo crecimiento vegetativo de la localidad, el cual, ha sido negativo desde 1991, produciéndose una pérdida poblacional, tomando como datos las defunciones y nacimientos, de 64 personas entre 1991 y 2005, habiéndose producido sólo los años 1998 y 2003 un incremento de población gracias al crecimiento vegetativo, registrándose ambos años un incremento de 3 individuos.

FIGURA 1: Evolución de la población en Fustiñana

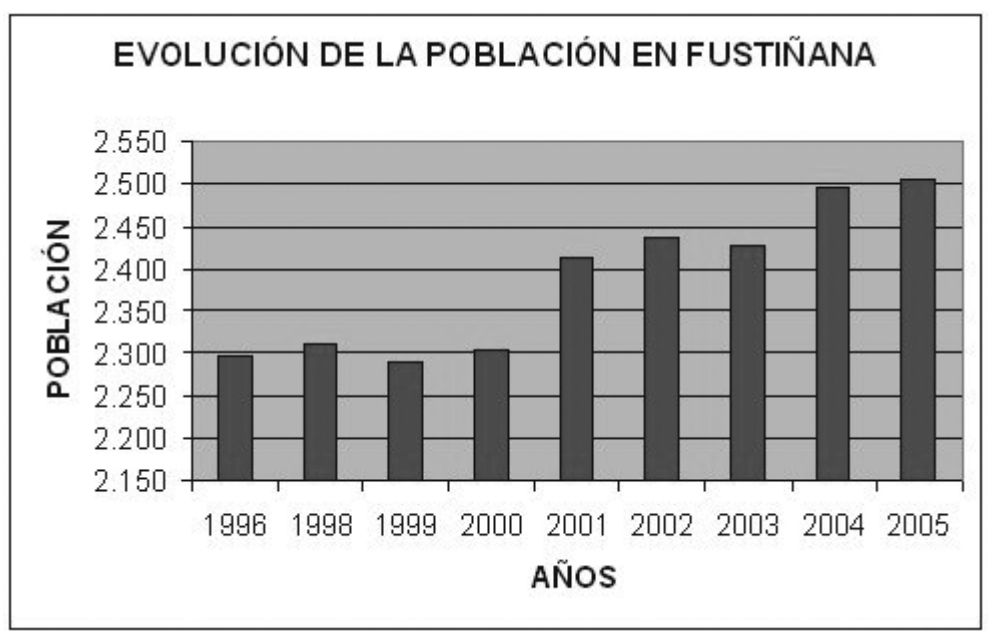


A partir del año 2000 este estancamiento demográfico sufre un vuelco y comienza la recuperación poblacional, como más arriba hemos indicado, gracias al fenómeno migratorio. El año 2000 la cifra total de vecinos en Fustiñana es de 2.304, pasando a suponer en 2005 un total de 2.506, lo cual supone un aumento de 202 personas en el quinquenio, con un $8,76 \%$ con respecto al total de 2000 .

Analizados los datos referentes al crecimiento vegetativo, las variaciones por años de la población y el espectacular aumento de la población de la Villa, nos damos una idea de la extraordinaria importancia que el fenómeno de la inmigración está teniendo para la reactivación demográfica y poblacional en la localidad. Esta importancia no sólo tiene que ver con el aumento poblacional en general, sino también con el rejuvenecimiento de una población envejecida.

TABLA 1: Evolución de la población en Fustiñana

\begin{tabular}{|cc|}
\hline años & población \\
1996 & 2.295 \\
1998 & 2.311 \\
1999 & 2.290 \\
2000 & 2.304 \\
2001 & 2.414 \\
2002 & 2.438 \\
2003 & 2.427 \\
2004 & 2.495 \\
2005 & 2.506 \\
\hline
\end{tabular}

En relación al aumento poblacional del Fustiñana, desde el año 1996 hasta el 2005, ha supuesto un incremento del $9 \%$, una cifra que está por debajo del 14\% del total del aumento para Navarra. Este porcentaje guarda relación con las tasas de crecimiento vegetativo de ambos campos de estudio, así, mientras Navarra va incrementando de manera exponencial su aumento de población gracias al mayor número de nacimientos que de defunciones, en Fustiñana ocurre lo contrario, presentando por este motivo un descenso de 37 individuos, al superar la mortalidad las cifras de natalidad. Teniendo en cuenta entonces la pérdida poblacional de la localidad en estos años en función del crecimiento vegetativo nos daremos cuenta de la importancia que tiene la inmigración, tanto exterior como interior, para el crecimiento demográfico de Fustiñana, de no haber sido por los movimientos migratorios la población actual seria de 2.250 vecinos.

TABLA 2: Movimiento natural de la población en Fustiñana

\begin{tabular}{|c|c|}
\hline AÑOS & MOVIMIENTO NATURAL DE LA POBLACIÓN \\
\hline 1996 & -2 \\
\hline 1997 & -16 \\
\hline 1998 & 3 \\
\hline 1999 & -5 \\
\hline 2000 & -5 \\
\hline 2001 & 0 \\
\hline 2002 & -4 \\
\hline 2003 & 3 \\
\hline 2004 & -2 \\
\hline $2005^{*}$ & -9 \\
\hline
\end{tabular}

TABLA 3: Evolución de la población en Navarra

\begin{tabular}{|c|c|c|c|c|c|c|c|c|}
\hline 1996 & 1998 & 1999 & 2000 & 2001 & 2002 & 2003 & 2004 & 2005 \\
\hline 520.574 & 530.819 & 538.009 & 543.757 & 556.263 & 569.628 & 578.210 & 584.734 & 593.472 \\
\hline
\end{tabular}

Tabla 4: Movimiento natural de la población en Navarra (\%)

\begin{tabular}{|c|c|c|c|c|c|c|c|c|c|}
\hline 1996 & 1997 & 1998 & 1999 & 2000 & 2001 & 2002 & 2003 & 2004 & 2005 \\
\hline 0,26 & 0,55 & 0,25 & 0,21 & 0,6 & 1,62 & 1,45 & 1,64 & 2,24 & 1,56 \\
\hline
\end{tabular}

\section{Evolución de la inmigración en Fustiñana.}

En cuanto al volumen de llegada a la localidad, el proceso, al igual que en Navarra, se ha realizado más tardiamente

FiguRA 2: Evolución de la población en Navarra

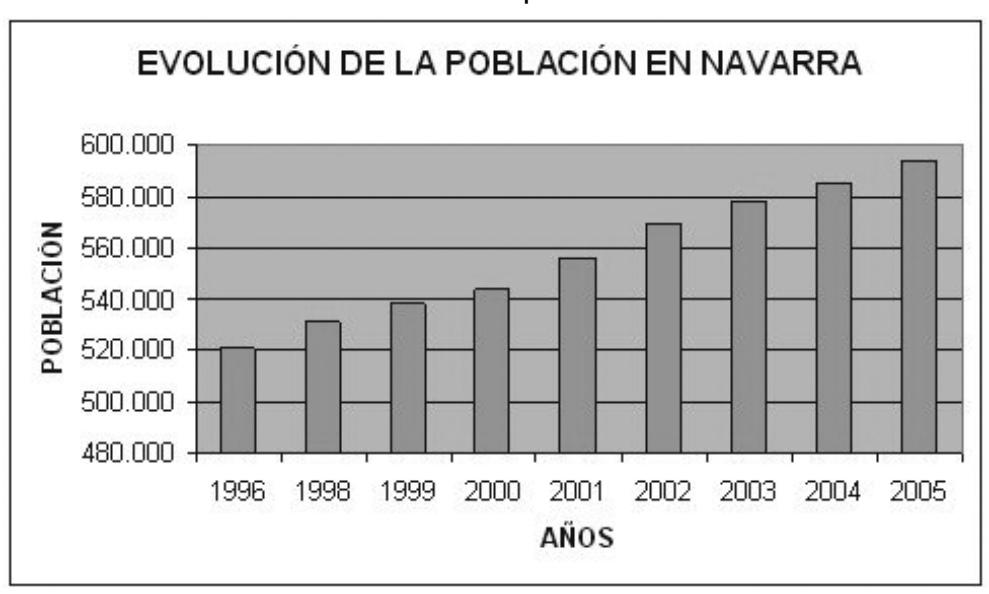


que en otras zonas de España, pero en los últimos años su intensidad es similar a la que se registra en la Comunidad.

Analizando el Padrón de 1996 nos da una cifra de 12 extranjeros residentes en Fustiñana, lejos de los 236 censados a fecha de 31 de julio de 2006, si nos fijamos en la cantidad total de inmigrantes llegados en los últimos diez años a la localidad obtendremos el siguiente porcentaje: la inmigración se ha incrementado en Fustiñana un 1.900\%. Si realizamos una comparativa con el mismo aumento que registro la población inmigrante en Navarra en el mismo periodo tenemos el siguiente porcentaje, un $1410 \%$. Esta comparativa nos muestra como en Fustiñana el aporte porcentual de población inmigrante es considerablemente mayor que en Navarra.

FiguRA 3: Evolución de la llegada de inmigrantes a Fustiñana

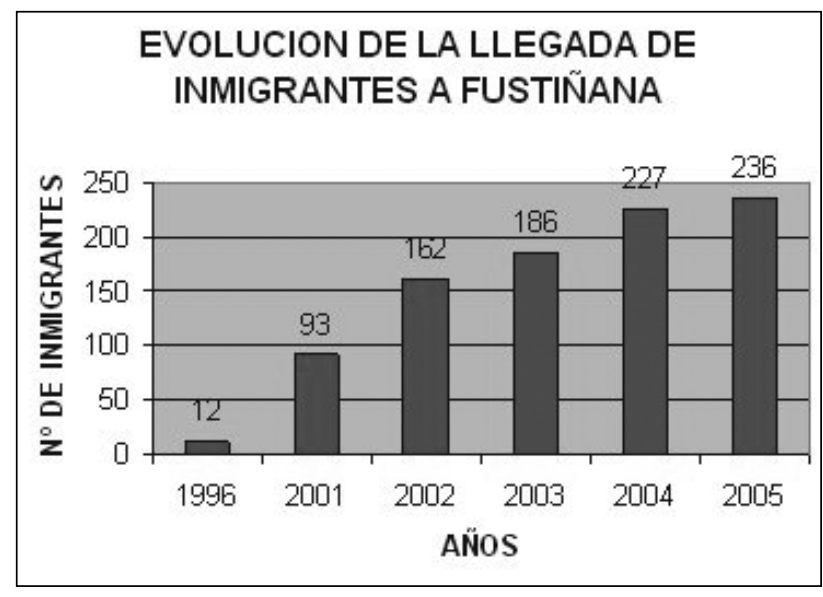

* Los datos de 2005 incluyen a todos los inmigrantes censados en Fustiñana a fecha de 31-7-2006.

FIGURA 4: Evolución de la llegada de inmigrantes a Navarra

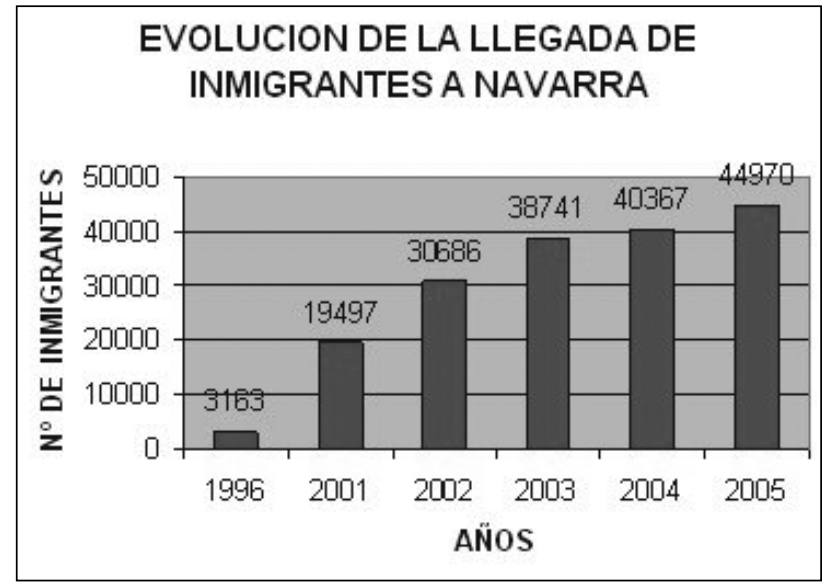

Estas diferencias en porcentajes están motivadas por la ubicación geográfica de Fustiñana, la cual está enclavada en el Valle Medio del Ebro, a escasa distancia de Tudela, ciudad con un extraordinario crecimiento económico, y sobre todo, con una próspera agricultura de frutales y hortalizas. La ocupación en labores agrícolas es el paso previo que dan los inmigrantes cuando Ilegan a España. Estos inmigrantes, una vez legalizada su situación personal, abandonan las labores agrícolas para mejorar sus expectativas pasándose al sector servicios, la construcción o el industrial.

Otro aspecto que ayuda a la presencia de inmigración en Fustiñana es el precio de la vivienda, tanto en propiedad como en alquiler, el cual es considerablemente más bajo que en las ciudades de su entorno. Este aspecto ayuda a entender la masiva presencia de extranjeros en una localidad la cual carece por completo de industria, además de no ser en absoluto un pueblo con un sector servicios desarrollado. En cuanto al mercado inmobiliario de alquiler, existe una gran oferta de casas antiguas de labranza que tienen unos precios bajos $y$, debido a su considerable tamaño, pueden ser ocupadas por un número considerable de personas sin que se den las situaciones de hacinamiento que se dan los pisos de las ciudades. En este punto, referente a la facilidad de encontrar una vivienda, hay que anotar que algunos agricultores facilitan a los inmigrantes que contratan alguna de sus viviendas antiguas las cuales ya no ocupan.

El tercer punto que ayuda a explicar estas diferencias es la facilidad de desplazamientos entre Fustiñana y Tudela, verdadero motor económico de la región. Las rápidas comunicaciones condicionan de manera positiva la presencia de inmigrantes en Fustiñana.

Por último hay que destacar el factor de agrupamiento que caracteriza a los inmigrantes, la nutrida presencia de la colonia búlgara es el más palmario ejemplo de este hecho.

\section{Composición por nacionalidad.}

La población extranjera empadronada en Fustiñana es mayoritariamente búlgara, este agrupamiento no es singular en Navarra, ya que parece existir cierta "especialización" entre los inmigrantes de las diferentes nacionalidades a la hora de elegir el destino final de su periplo, habiéndose elegido entre los inmigrantes búlgaros la localidad de Fustiñana, al igual que Cintruénigo lo es para los ecuatorianos o Tudela para los magrebies. Los búlgaros censados en Fustiñana son 122 , lo cual representa más del $50 \%$ del total de inmigrantes asentados en la localidad. Como decíamos más arriba, la solidaridad entre los inmigrantes, además de la buena acogida de este colectivo entre el resto de vecinos de la Villa, unida a las condiciones de vivienda y económicas comentadas en el punto anterior, ayudan a entender esta masificación de ciudadanos de una misma nacionalidad en Fustiñana.

Si es sorprendente la masiva presencia de búlgaros en este pueblo, no lo es menos la nula presencia de ciudadanos rumanos, los cuales representaban en Navarra en 2003 un $5 \%$ de la totalidad de los inmigrantes, dos puntos por encima del 3\% que tenían los búlgaros. Esta ausencia de ciudadanos rumanos refuerza la teoría de agrupamiento de las diversas colonias de inmigrantes en localidades diferentes, en 
las cuales una de ellas ostenta de manera clara la hegemonía entre el heterogéneo colectivo de inmigrantes.

El segundo grupo lo componen los latinoamericanos, con 61 individuos, entre los que destacan los 45 ciudadanos ecuatorianos. Como se comprueba en los gráficos este colectivo es el más numeroso en Navarra, con un 55\% sobre el total. En Fustiñana los latinoamericanos representan el $25,8 \%$ de total de inmigrantes, lejos por lo tanto de la cifra total en Navarra.

Figura 5: Porcentaje de inmigrantes en Fustiñana según nacionalidad de origen

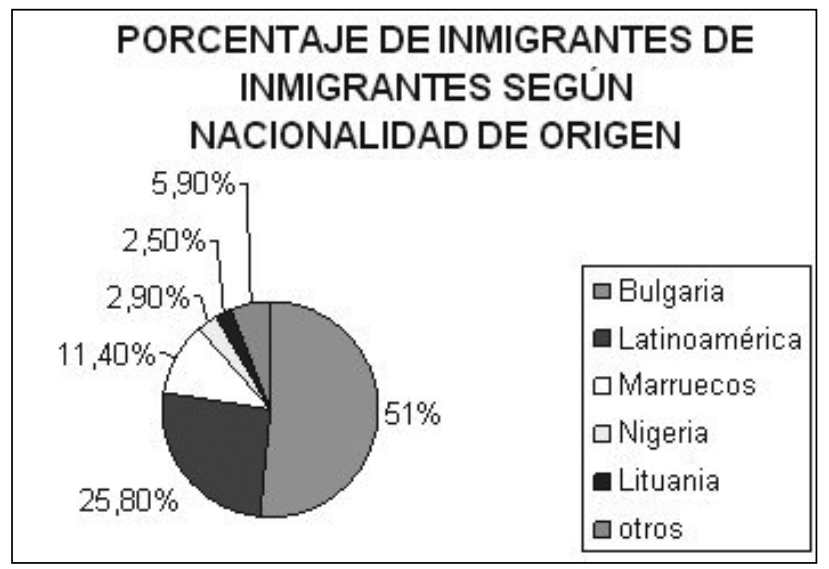

Figura 6: Porcentaje de inmigrantes en Navarra según nacionalidad de origen

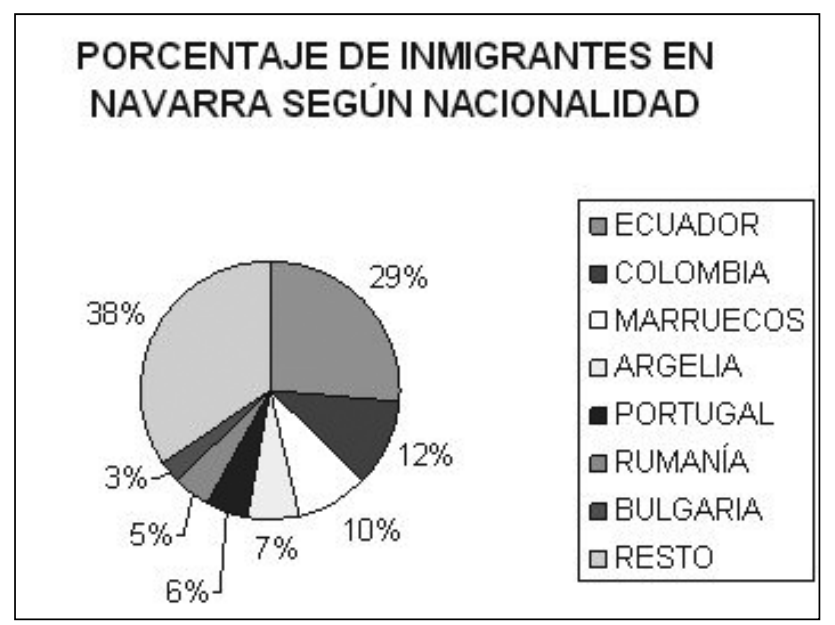

En tercer lugar se sitúan los ciudadanos magrebies, con un total de 28 personas. En este grupo la presencia de argelinos es meramente testimonial, tan sólo uno de ellos posee esta nacionalidad. En cuanto a los inmigrantes magrebies es también interesante que presentan una cifra muy baja en comparación con otros pueblos de la Ribera, zona de Navarra donde de ubica el mayor porcentaje de individuos de dicha procedencia. La menor presencia de magrebies en Fustiñana se debe a la inexistencia de cultivos de frutales y viñedos, los cuales precisan de gran cantidad de mano de obra en la recogida del fruto, además de la mecanización de labores como la recogida del tomate, la cual empleaba hasta hace muy pocos años a una gran cantidad de ciudadanos norteafricanos en los meses de agosto y septiembre.

El resto de inmigrantes lo componen 27 individuos de 15 nacionalidades diferentes.

\section{Estructura por sexo:}

La población inmigrante residente en Fustiñana presenta un índice de masculinidad del $52,9 \%$, con una cifra total de 124 hombres. El número de mujeres es de 112, dándose de este modo un menor índice de masculinidad que en el conjunto de la Comunidad Foral, en la cual, a fecha de 2003, habia 125 hombres por cada cien mujeres. Realizando una comparativa con el índice de masculinidad de la población autóctona en el municipio nos damos cuenta que entre los nativos este índice es menor, en concreto presenta una proporción del 50,2\%, con un total de 1.160 hombres por 1.150 mujeres. Estos datos nos confirman la teoría que argumenta que emigran más los hombres que las mujeres, pero la diferencia en este caso es tan pequeña que esta máxima está dejando de ser un dogma entre los estudiosos de los comportamientos migratorios.

Realizando un análisis entre los principales colectivos, agrupados por paises de procedencia, obtenemos los siguientes resultados.

- Entre la colonia búlgara el índice de masculinidad es del $54,09 \%$, cifra algo más alta que la correspondiente a la población inmigrante en su conjunto, lo cual indica que emigran más los búlgaros que las búlgaras.

- Los inmigrantes ecuatorianos poseen un índice de masculinidad del 53,3\%, con una cifra total de 24 hombres por 21 mujeres. Como en el caso anterior, estamos en una proporción que supera la del conjunto inmigrante.

- La inmigración marroquí tiene un índice de masculinidad del $62,9 \%$. Este predominio masculino se da en todos los lugares donde se asienta este colectivo. Las principales razones de esta abrumadora mayoría masculina son principalmente de tipo cultural.

- Es en el resto de los colectivos de inmigrantes de otras nacionalidades lo que provoca el descenso del índice de masculinidad, destacando el caso de Colombia. En este caso hay 4 mujeres por un sólo hombre. Los inmigrantes de los demás países que presentan mayoría femenina, son tan poco numerosos que sus datos, analizados porcentualmente, no reflejarían una situación general, ya que en muchos de estos colectivos las diferencias son de un solo miembro, de lo cual no se pueden extraer conclusiones generales. 


\section{Estructura por edades:}

A través de los datos conseguidos del Ayuntamiento, se ha establecido la siguiente estructura por edades de la población inmigrante en Fustiñana. Los datos aportados sólo diferencian a los mayores de 16 años de los menores de esta edad, así como la nacionalidad de los menores.

De los 236 inmigrantes censados a fecha de 31-7-06, 45 de ellos poseen una edad comprendida entre los cero y dieciséis años, lo cual supone un 19,06\%, como podemos comprobar por encima de la media en Navarra, la cual estaba situada para este tramo de edad (fuentes de 2003) en el 15,6\%.

El resto de los inmigrantes superan esa edad y, a pesar de no contar con datos oficiales, prácticamente el cien por cien de ellos están situados entre los 17 y los 45 años de edad, superando tan sólo ese tramo de edad dos personas mayores que acompañan a sus familias para el cuidado de sus nietos. Como vemos estamos ante una población muy joven y en edad laboral, lo cual repercute en el descenso del índice de envejecimiento de Fustiñana. Un dato indirecto que nos sitúa ante la juventud de esta población es la ausencia de mortalidad. Desde que se inició la llegada de inmigrantes a la localidad no se ha producido ni un solo fallecimiento, circunstancia que seria imposible de darse en una sociedad compuesta por individuos que abarcasen todos los tramos de edad que forman la composición de cualquier estudio demográfico.

\section{Natalidad.}

En cuanto a la natalidad, hasta el año 2002 no hay registrado ningún nacimiento de hijos de madre extranjera. Desde este año hasta el 31 de julio de 2006 se han producido un total de ocho nacimientos.

Es preciso anotar que el primer nacimiento se produce a finales de 2002, cuando el proceso de llegada de inmigrantes ya estaba consolidado y presentaba unas cifras considerables. Esta circunstancia se produce debido al proceso de asentamiento de la población, es decir, no se plantean la necesidad de la paternidad debido a las difíciles condiciones de su aclimatación a nuestra sociedad, a la ausencia de un primer trabajo estable, a la llegada en primer lugar de la población masculina, la cual acarrea a la familia una vez asentada y, debido al predominio de población búlgara en Fustiñana, al comportamiento demográfico de este colectivo, el cual es similar al de los países de la Europa occidental.

\section{INMIGRACIÓN Y MERCADO LABORAL.}

\section{Introducción.}

En un pasaje de este trabajo se comentaba el dato de la falta de industria en Fustiñana. Esta ausencia del Sector Secundario, tan sólo existe una fábrica con unos diez emplea- dos, se presentaba como un dato que en principio no debería haber ayudado a la llegada de inmigrantes a la localidad, sin embargo los datos sobre inmigrantes censados ponen en entredicho el tradicional binomio de falta de una economía industrial y la ausencia de inmigración. Además se ha comentado que el sector servicios no estaba demasiado desarrollado, tan sólo los bares y tiendas se dedican a este sector.

Una vez dicho esto queda la pregunta formulada, ¿a qué se dedican los inmigrantes residentes en Fustiñana?, ¿dónde trabajan?

Los datos que reflejan este estudio han sido conseguidos mediante entrevistas personales a aquellos agricultores, empresas de construcción, almacenes de transformación de hortalizas y personas que emplean a inmigrantes en el servicio doméstico ylo en el cuidado de ancianos, además de entrevistas a numerosos inmigrantes que trabajan fuera del municipio.

\section{Impacto en el mercado laboral:}

Analizando la ocupación de los inmigrantes por los diferentes sectores de actividad obtenemos los siguientes resultados:

\section{$1^{\circ}$. Sector primario:}

La agricultura es, junto a la construcción, el primer empleo que ocupan los inmigrantes llegados a Fustiñana. Estos se ocupan de manera temporal, aún así, los meses de trabajo al año suelen superar los seis, repartidos entre las diferentes estaciones del año. Encuestados los empresarios agrarios sobre los puestos de trabajo que ofertan y quienes los ocupan obtenemos los diferentes resultados:

El número total de individuos que trabajan en este sector es de 24. Analizando esta cifra por sexo y nacionalidad presentan estas características:

El número de búlgaros es de 20 trabajadores, representando el $83,3 \%$ del total de empleados agrarios. Los cuatro restantes son de nacionalidad marroquí, trabajadores de la misma explotación y los más antiguos en este trabajo.

Entre los se dedican a trabajar en este sector hay que diferenciar entre empleados, aquéllos que como hemos dicho trabajan de manera continua, y los temporeros, aquéllos que se ocupan tan sólo durante un corto periodo de tiempo para realizar un trabajo y, una vez finalizado, vuelven a su lugar de residencia. En cuanto a estos temporeros es necesario anotar la existencia de Bolsas de Trabajo en los sindicatos agrarios, los cuales gestionan de manera directa las contrataciones de estas personas por los agricultores que precisan sus servicios. Estos temporeros, cuando están realizando su trabajo, no se han contabilizado para el presente trabajo, aún así creemos que es preciso anotarlo como una circunstancia que, coyunturalmente, provoca una masiva presencia de trabajadores extranjeros en el campo fustiñanense. 
En cuanto a su estructura por sexo, sólo se emplean en la agricultura cuatro mujeres, las cuales son en su totalidad de nacionalidad búlgara.

Como conclusión al empleo sobre el sector primario diremos que sorprende la nula presencia de trabajadores latinoamericanos a pesar de ser el segundo grupo en importancia entre el colectivo inmigrante. Esta circunstancia se da por la negativa de los agricultores a su contratación, ya que, según su opinión, no están lo suficientemente preparados para sobrellevar las duras tareas agrarias.

La masiva presencia de ciudadanos búlgaros, muy por encima de su representación sobre el total de la población inmigrante, está motivada por la integración de este colectivo en la sociedad de Fustiñana, por la necesidad de contratos por parte de los balcánicos para legalizar su situación y por el rendimiento que ofrecen a la persona que precisa de sus servicios.

Los trabajadores marroquies están en minoría en este sector. Este dato es sorprendente ya que fue el primer colectivo que desarrolló las tareas de este tipo, dándose la circunstancia que en la vecina ciudad de Tudela y en el pueblo de Ribaforada son el principal colectivo que se dedica a la agricultura. La situación de inferioridad que tienen en Fustiñana está motivada por que la colonia búlgara los ha ido desplazando, además de por la reticencia a contratarlos por los agricultores debido a las diferencias socio-culturales entre ambas comunidades, la magrebi y la autóctona, y por la existencia de antiguos conflictos entre contratantes y contratados.

\section{Sector secundario.}

En este estudio se ha comentado la inexistencia del Sector Secundario en Fustiñana. Esta circunstancia debe ser matizada porque hay en la localidad tres empresas que se dedican a las labores de transformación y envasado de los productos agrícolas, en concreto a las hortalizas. Para este trabajo se han incluido en el sector secundario, aunque la cotización a la Seguridad Social es, en todas las empresas, las del Régimen Especial Agrario, con lo cual estamos a caballo entre el Sector Secundario, en realidad los trabadores realizan trabajos mecánicos y en cadena, y el Primario, tanto por los productos que elaboran (en ningún caso se produce una transformación total del producto como el enlatado, congelado, etc.) como por su régimen de cotización agraria.

En este sector industrial se han contabilizado 14 personas. De estas dos de ellas trabajan en empresas ubicadas fuera de la Villa En los almacenes de transformación trabajan las doce restantes, repartiéndose, en función de nacionalidad y sexo de este modo:

El predominio en estas labores es absoluto para las mujeres, de los doce empleos diez los ocupan las mujeres. Esta circunstancia está asociada a las labores realizadas como son el pelado de cebolla, el troceado de bróculi, coliflor y alcachofa y el envasado en bandejas para su venta en el mercado de productos frescos. Esta proporción se da también en los empleados autóctonos del sector. En cuanto a su nacionalidad, seis de ellas son búlgaras, tres ecuatorianas y la restante marroquí.

La nacionalidad de los trabajadores masculinos está más equilibrada, con la presencia de un búlgaro y un ecuatoriano.

El predomino masivo de trabajadoras búlgaras responde, en líneas generales, a las mismas razones de su predominio en el sector agrario, esto es, aceptación en la sociedad, trabajo realizado con profesionalidad, etc. En este sector, y respecto al empleo femenino, se reflejan las mismas situaciones socio-culturales que en los países de origen, nos estamos refiriendo a la escasa participación que la mujer magrebí posee en el mercado de trabajo.

\section{Construcción:}

El sector de la construcción posee una gran pujanza en Fustiñana. Al igual que en el resto de España, este sector está teniendo un desarrollo extraordinario. El gran auge inmobiliario existente en el país ha llegado incluso a las más pequeñas localidades. En Fustiñana este desarrollo ha provocado la extensión de su perímetro urbano, además de un desarrollo en su interior al ser derribadas antiguas edificaciones que en su día se utilizaron como almacenes agrícolas y corrales de ganado. Esta fiebre constructiva tiene que ver, además de con la especulación urbanística, con la llegada de nuevos habitantes procedentes de la inmigración, los cuales necesitan un techo para vivir.

Este desarrollo de la construcción ha motivado un aumento de las empresas constructoras de la localidad, en concreto se contabilizan seis de ellas. La dureza de las tareas de albañilería y las mejores oportunidades que tanto la industria como los servicios ofrecen a los trabajadores, han tenido como consecuencia el abandono por parte de los empleados autóctonos de este tipo de empleos, siendo llenado este vacío por los inmigrantes.

Como más arriba mencionábamos, la agricultura y la construcción son los primeros sectores donde se emplea la población inmigrantes. En el caso concreto de Fustiñana, en este sector trabajan, para las empresas locales, una cantidad de 15 inmigrantes, todos ellos masculinos. Su distribución por nacionalidades y porcentajes es la siguiente:

- 13 latinoamericanos (11 ecuatorianos y 2 bolivianos), que suponen el $86,6 \%$ del total.

- 2 ciudadanos búlgaros, con el 13,4\% restante.

Deteniéndonos en estos datos extraemos las siguientes conclusiones: en este sector el predominio de los latinoamericanos es total. Si la población total masculina de latinoamericanos en edad de trabajar es de 22 individuos, nos da- 
mos cuenta de la importante participación de este colectivo en las labores de la construcción ya que se dedican a ella el $59,9 \%$, y este porcentaje sólo para los que trabajan en la localidad, siendo conscientes que alguno de ellos realiza labores de albañilería en la vecina Tudela.

El caso de los trabajadores búlgaros también es especial, dándose una pequeña proporción en este sector, al contrario de lo que ocurría en el agrario. Este hecho se explica en parte por la posibilidad de promoción en otros sectores que estos inmigrantes poseen

En cuanto a los inmigrantes magrebies es notoria su ausencia, lo cual fortalece la tendencia de especialización de este colectivo humano en el sector primario.

\section{Sector servicios.}

Este sector, a pesar de estar poco desarrollado en Fustiñana, ocupa en total a 23 personas.

\section{EMPLEO DOMÉSTICO:}

En los servicios hemos incluido el trabajo doméstico y el cuidado de ancianos, siendo conscientes que la totalidad de personas que trabajan en estas labores están fuera del sistema de cotización al la Seguridad Social y que, por lo tanto, no tienen la posibilidad de salir reflejados como trabajadores en ningún organismo oficial.

En las labores de empleadas domésticas poseen la hegemonía las ciudadanas latinoamericanas con ocho de ellas dedicadas a estas labores, el resto de las 3 personas dedicadas a este trabajo son de nacionalidad búlgara.

FIGURA 7: Proporción de la ocupación laboral de los inmigrantes según sexo

\section{PROPORCIÓN DE LA OCUPACIÓN LABORAL DE LOS INMIGRANTES SEGÚN SEXO}

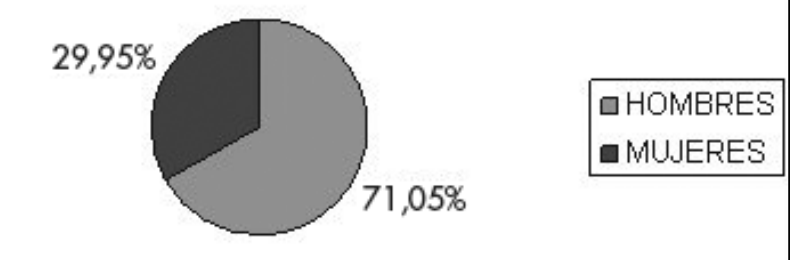

Como vemos, también en estos trabajos existe una gran diferencia entre quienes lo realizan en función de su nacionalidad, predominando netamente las mujeres latinas. Las causas que ayudan a explicar esto son las siguientes: en primer lugar el conocimiento del idioma. Para muchas familias $y$, sobre todo ancianos, es fundamental que aquél que esté en su casa se pueda comunicar con él. En segundo lu- gar la especialización de las mujeres latinas en estas labores. En España se ha convenido tácitamente que las latinas realicen estas labores, estando el mercado saturado de ciudadanas sudamericanas que se ofrecen para este tipo de labores. Por último, otra de las causas es la mejor preparación técnica y académica que poseen las búlgaras, lo cual les lleva a buscar unos trabajos de mayor calidad y rendimiento económico. En este aspecto, la presencia de trabajadores búlgaros en puestos de mayores ingresos económicos, libera en parte a sus esposas de las ingratas tareas del servicio doméstico.

FIGURA 8: Distribución de los trabajadores inmigrantes en la industria según sexo

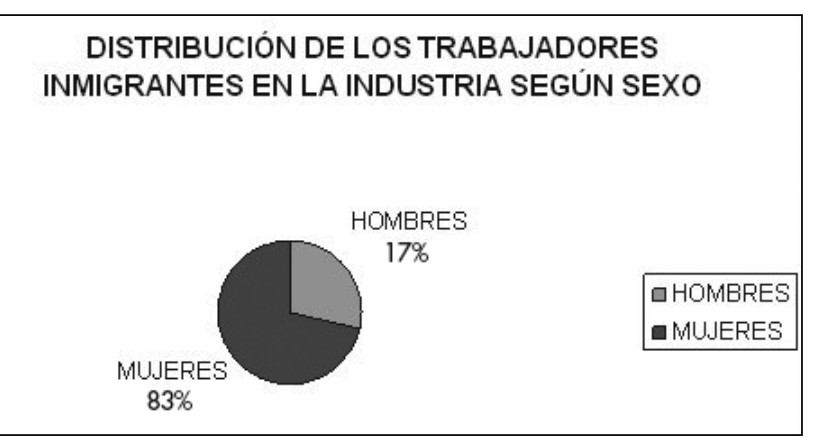

FIGURA 9: Ocupación de los inmigrantes según sectores de actividad

OCUPACIÓN DE LOS INMIGRANTES SEGÚN SECTORES DE ACTIVDAD

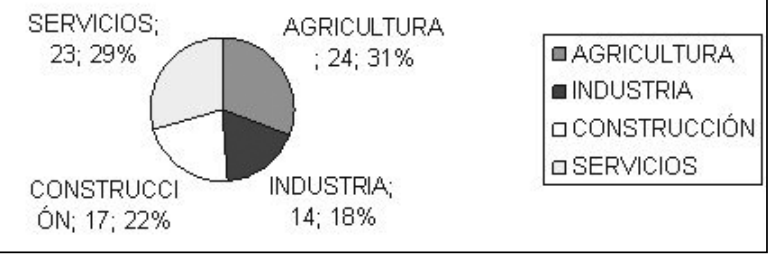

En este apartado tampoco aparece ninguna trabajadora magrebí, siendo el sector servicios, en general, el más reticente a contratar a personas de este origen, por razones tanto culturales como sociales y étnicas.

\section{TRANSPORTE:}

El transporte por carretera es otro de los trabajos de gran dureza que los autóctonos poco a poco van dejando de cubrir. En Fustiñana se dedican a las labores de conducción de camiones diez individuos, todos ellos de nacionalidad búlgara. El predominio de los búlgaros en el transporte vemos que es total, aunque en este sector las condiciones de trabajo son de gran dureza, los ingresos salariales que les proporciona están por encima de los que cobrarían en otro trabajo que no exija ninguna especialidad. 
OTROS:

En esta apartado incluiremos el resto de ocupaciones que abarcan el extenso sector servicios.

La hostelería y el comercio tan sólo ocupan a una persona.

Los Servicios Sociales de Base tienen contratada a una persona a través de sus programas de acogida.

\section{Metodologia:}

Para la elaboración del presente trabajo, a falta de los datos totales, hemos realizado un muestreo con 78 inmigrantes trabajadores, sobre un total de 191 en edad laboral. Ante la falta de datos sobre la población inactiva que se encuentra dentro de los grupos de edad laboral, y aplicando los porcentajes de inactividad que existian en Navarra en el estudio de 2003, esta cifra se reduce en 15 personas, situándose las personas inmigrantes que trabajan en unas 176.

Una de las conclusiones que se desprenden del presente trabajo es que se da la misma circunstancia que a nivel de Navarra, siendo dicha circunstancia la especialización de los diferentes colectivos de inmigrantes, por nacionalidades, en los diferentes sectores de la actividad. No estando dicha especialización, al menos de manera determinante, motivada por una mayor preparación técnica o académica de los inmigrantes, que si la hay en caso de los búlgaros, sino por circunstancias de tipo étnico-cultural y por prejuicios sociales y diferencias idiomáticas, así como por las reticencias de los mismos grupos de inmigrantes a la hora de trabajar en alguno de los sectores.

Otro aspecto es la masiva presencia de inmigrantes en trabajos que no requieren, a priori, ninguna especialización técnica, estando ocupando trabajos de peones y empleados sin especialidad.

\section{EL IMPACTO DE LA INMIGRACIÓN EN EL SECTOR INMOBILIARIO.}

Como se viene comentando desde el inicio de este trabajo, la llegada de inmigrantes precisa de una infraestructura de viviendas que los acoja. Si estos inmigrantes tienen una necesidad imperiosa, esta es la vivienda, siendo el tema de la vivienda es un asunto de gran trascendencia para ellos. El aumento de la población de Fustiñana en casi un 10\% en los últimos años ha provocado un auge constructivo. Vemos entonces como este aporte poblacional ha incidido, de manera directa o indirecta, en el gran aumento de viviendas que se está dando en Fustiñana en estos últimos años. Este auge del mercado inmobiliario local ha provocado que antiguas viviendas vacias, en algunos casos en franco estado de abandono, se hayan incorporado a este mercado, hecho este de gran importancia, ya que, ha tenido como consecuencia
FIGURA 10: Ocupación de los inmigrantes en la agricultura

\section{OCUPACIÓN DE LOS INMIGRANTES EN LA AGRICULTURA}

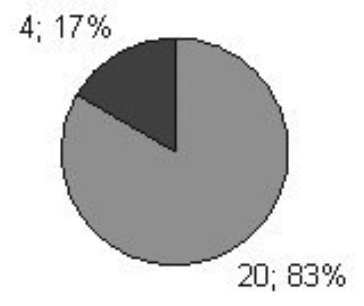

口BÚLGAROS

a MARROQUÍES

FIGURA 11: Ocupación de los inmigrantes en la industria según nacionalidad

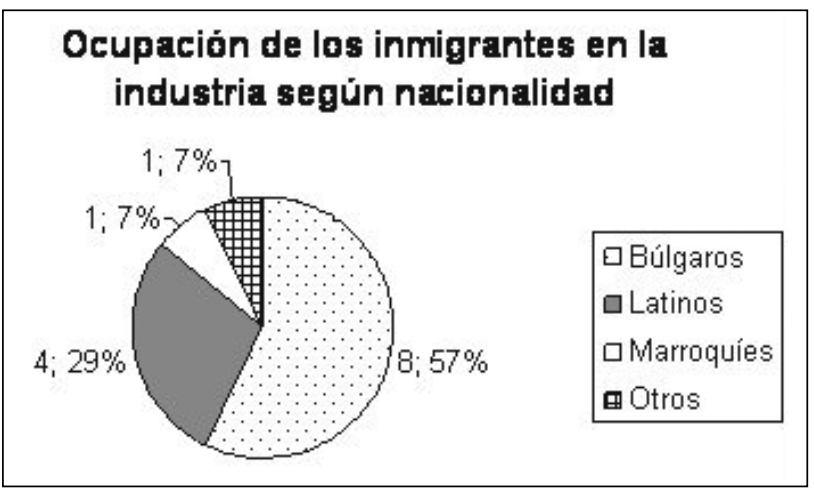

FIGURA 12: Ocupación de los inmigrantes en la construcción

\section{OCUPACION DE LOS INMIGRANTES EN LA CONSTRUCCIÓN}

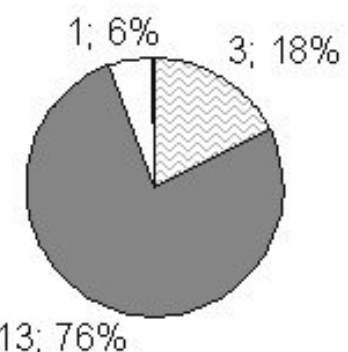

FiguRA 13: Ocupación de los inmigrantes por actividad en el sector servicios

\section{OCUPACIÓN DE LOS INMGRANTES POR ACTIVIDAD EN EL SECTOR SERVICIOS}

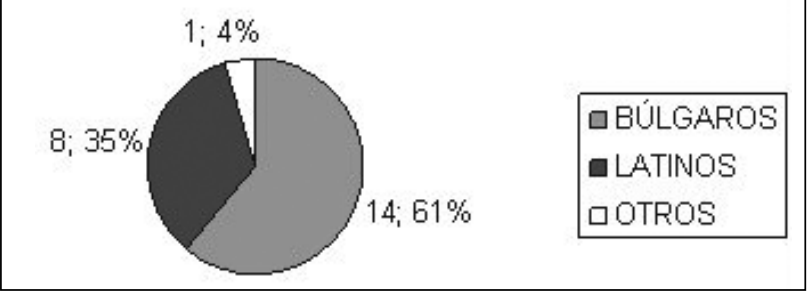


un crecimiento por "implosión» de la superficie de Fustiñana. La ocupación por parte de los inmigrantes de este tipo de viviendas, así como la rehabilitación de antiguas en estado casi de derrumbe, ha impedido que el pueblo se haya extendido demasiado por medio de nuevas urbanizaciones y barrios, contribuyendo la inmigración, a través de sus necesidades de vivienda, a la conservación del interior de una población que en algunos puntos estaba en franco estado de abandono.

FIGURA 14: Utilización de la vivienda en función del régimen de tenencia

\section{UTILIZACIÓN DE LA VIVIENDA EN FUNCIÓN DEL RÉGIMEN DE TENENCIA}
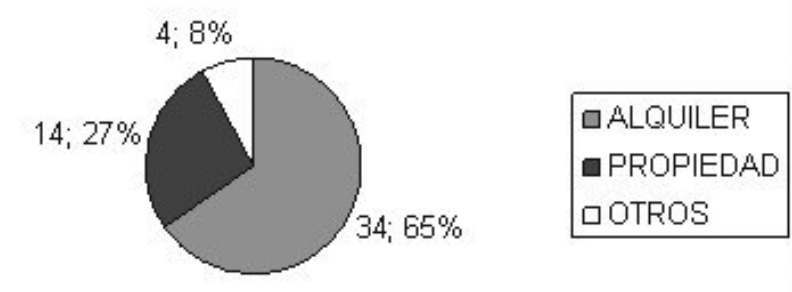

Una circunstancia que se da en el tema de la vivienda de alquiler es que la ocupación de estas viejas viviendas van siendo habitadas de manera rotativa por los nuevos inmigrantes que van llegando, así, cuando una familia lleva el suficiente tiempo residiendo en Fustiñana y sus recursos económicos se lo permiten, las abandonan por otras de mayor calidad o por la compra de una vivienda nueva. Estas viviendas antiguas, una vez desocupadas por estos inmigrantes, enseguida son utilizadas por los recién incorporados, estando de este modo continuamente ocupadas. Como dato adicional diremos que si una vivienda es ocupada, por ejemplo, por inmigrantes ecuatorianos, los siguientes que las ocupan son nuevamente ecuatorianos, dándose de este modo una ocupación continua de la misma vivienda por diferentes familias de la misma nacionalidad. Esta circunstancia nos lleva a sospechar que muchos inmigrantes llegan a residir a Fustiñana, procedentes de su país o de otra localidad cercana, cuando tienen asegurada la vivienda libre, al estar permanentemente en contacto los inmigrantes que residen en la localidad con aquéllos que también pretenden venir a vivir a Fustiñana.

\section{Metodología:}

Se han utilizado como fuentes los datos del Censo de Viviendas de 1996,2000 y 2001, proporcionados por el INE y por el Instituto Navarro de Estadística.
Se ha realizado un recuento por viviendas, unidad por unidad, de todas aquellas en las que residen inmigrantes.

\section{Evolución del número de viviendas en Fustiñana:}

Según los datos aportados por el Instituto de Estadística de Navarra, el número de viviendas en Fustiñana ha seguido la siguiente evolución.

- 1996: 854 viviendas.

- 2000: 952 viviendas.

- 2001: 972 viviendas.

Estos datos incluyen todas las viviendas del municipio, debido a que el Censo de Viviendas se elabora cada diez años, y el último se elaboró en 1996, los datos de 2000 y 2001 aunque son completos en cuanto al número total de viviendas, no lo son a su reparto por vacias u ocupadas. Para el resto de la serie hasta 2006, ni el Ayuntamiento ni la Administración Autonómica han proporcionado dato alguno. En cuanto al Censo de 1996, éste nos arroja un número de viviendas vacias de 176 , que equivale al $20 \%$ del total de viviendas. En el año 1996 todavía no había comenzado el proceso de llegada de inmigrantes a Fustiñana, con lo cual este importante número de viviendas vacias, ha sido imprescindible para dar alojamiento a los sucesivos inmigrantes que han ido llegando desde esta fecha hasta la actualidad.

\section{Régimen de tenencia de la vivienda por los inmigrantes:}

PROPORCIÓN DE OCUPACIÓN DE VIVIENDAS EN FUNCIÓN DEL RÉGIMEN DE TENENCIA:

\section{ALQUILER:}

El total de viviendas en régimen de alquiler es de $34 \mathrm{vi}-$ viendas, lo cual supone el 65,3\% del total de las 52 viviendas ocupadas, de una manera u otra por los inmigrantes. Estos alquileres están distribuidos por nacionalidades de la siguiente manera:

FIGURA 15: Ocupación de viviendas por nacionalidades en régimen de alquiler

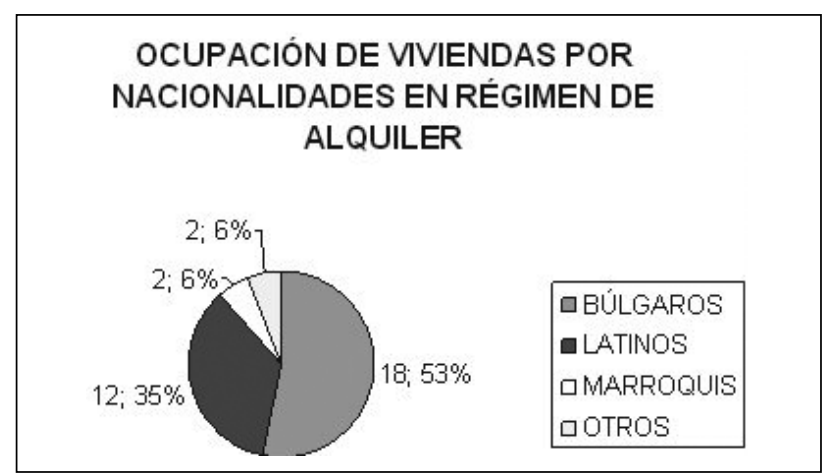




\section{PROPIEDAD:}

En cuanto a la propiedad el reparto por nacionalidades es el siguiente:

FIGURA 16: Ocupación de viviendas en propiedad según nacionalidades

\section{OCUPACIÓN DE VIVIENDAS EN PROPIEDAD SEGÜN NACIONALIDADES}

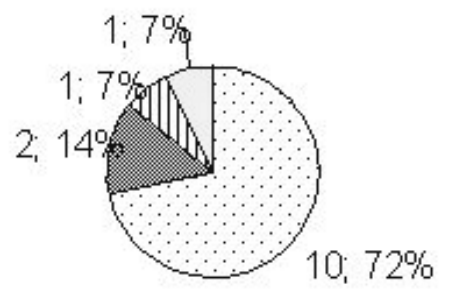

\begin{tabular}{l}
$\because$ BULGARO \\
⿴囗大 ATINOS \\
M MARROQUIS \\
$\square O T R O S$ \\
\hline
\end{tabular}

OTROS:

En este apartado se incluyen los inmigrantes que están ocupando viviendas en casos especiales que no son ni alquileres ni propiedad.

Se han constatado la existencia de dos familias de ecuatorianos que conviven con autóctonos debido a la relación sentimental de un miembro de la citada familia con el vecino en cuestión.

Otra familia de ecuatorianos convive en la vivienda con una persona mayor a la cual atienden, siendo la utilización de dicha vivienda una de las formas de pago por los servicios prestados a dicha persona.

Por último se ha constatado la presencia de un inmigrante de nacionalidad polaca residiendo en precario en una caseta de huerto.

\section{Conclusiones:}

Según este estudio, el reparto de inmigrantes por unidad de vivienda es de 4,5 personas por hogar. Como podemos comprobar, la existencia de un importante mercado de alquiler de viviendas, así como sus asequibles precios, han tenido como consecuencia la ausencia de masificación de personas en al utilización de las viviendas.

Si el régimen de alquiler es el más característico de los inmigrantes, es interesante subrayar la importancia que está adquiriendo el régimen de propiedad, representando el 27\% del total de las viviendas ocupadas por los inmigrantes. De entre todos ellos cabe destacar el peso de los búlgaros, los cuales con un total de 14 viviendas en propiedad, las cuales representan el 35,7\% del total de las viviendas que ocupan, encabezan esta forma de tenencia de la vivienda.
La importancia que está adquiriendo entre los inmigrantes éste régimen de tenencia nos da una idea de las pretensiones de continuidad que tienen respecto a su definitivo asentamiento en Fustiñana. El razonable precio de la vivienda nueva en la localidad, unido a las ayudas recibidas por la Administración para la compra de esta, también ayudan a entender el paso del régimen de alquiler al de propiedad. En cuanto al colectivo búlgaro, su carácter de europeos les otorga una mentalidad cercana a la nuestra, en la cual está muy arraigada la cultura de poseer una vivienda propia.

\section{CONCLUSIONES.}

La inmigración en Fustiñana es un fenómeno nuevo en este municipio. Este hecho está alterando de manera significativa las características poblacionales de la Villa. La nueva coyuntura económica nacional, unida a las grandes diferencias en cuanto a riqueza que separan al llamado Primer Mundo del Tercer Mundo, está generando el desplazamiento de grandes flujos de población desde los paises pobres hacia los ricos, a los cuales hay que sumar los miles que cada año acuden a nuestro país desde los antiguos países comunistas del Este europeo.

Dentro de este amplio contexto internacional es donde hay que ubicar la llegada de inmigrantes a cualquier punto de la geografía nacional, ya que, la masiva llegada de inmigrantes, está teniendo como consecuencia que éstos se repartan por todo el solar español

Analizando las peculiaridades económicas de Fustiñana, sobre todo la ausencia de unos sectores secundarios y terciarios desarrollados, obtenemos como resultado que las tradicionales leyes que intentaban explicar los factores de atracción que movían a los flujos migratorios hacia los nuevos destinos de asentamiento, se han quedado obsoletas, cuando no inservibles totalmente. En el caso analizado hemos comprobado como una pequeña población sin apenas recursos laborales propios, se convierte en un núcleo receptor de inmigrantes, ya que, las nuevas circunstancias económicas y de empleo, ofrecen nuevas posibilidades de ocupación y de mejora económica.

Como hemos comprobado en nuestro estudio, este fenómeno migratorio no sólo es beneficioso para la población inmigrante, subsidiariamente la población de acogida se beneficia en grado máximo de él. El aporte humano foráneo está sirviendo para reactivar la economía en poblaciones que se encontraban en franca decadencia, además, está teniendo como consecuencia la modificación radical del comportamiento demográfico de estos pueblos de acogida, los cuales estaban en constante decadencia demográfica, con una población envejecida y un crecimiento vegetativo en muchas ocasiones negativo.

En cuanto a la economía de las pequeñas poblaciones que acogen a estos colectivos de inmigrantes, hemos com- 
probado que producen una mejora en su conjunto, siendo el sector de la construcción, con las nuevas necesidades de alojamiento que la inmigración plantea, el mayor beneficiado de los diferentes sectores económicos.

Otro sector que se ha visto favorecido por la llegada de nuevos contingentes de población es la agricultura. El abandono de los trabajos agrícolas por parte de la población autóctona hubiese tenido como consecuencia la pérdida de la pujanza que los cultivos hortofrutícolas estaban teniendo en el sector agrario. El trabajo de los inmigrantes en el campo ha compensado la falta de mano de obra tradicional.

Por otra parte, y teniendo en cuenta que la inmigración también es una población con necesidades de consumo, ha permitido que el pequeño sector servicios de localidades como Fustiñana adquiera un nuevo impulso y supere la crisis a la cual estaba sometido debido a la regresión demográfica de estas poblaciones y al envejecimiento de sus gentes.

De entre las consecuencias sociales más importantes hay que destacar el cuidado de ancianos y niños por parte de la población inmigrante femenina. La dedicación de las mujeres de otras nacionalidades a estas tareas ha supuesto el aumento de la calidad de vida en estos sectores poblacionales los cuales estaban siendo abocados a un progresivo abandono por parte de sus familias y la sociedad en su conjunto.

Por último hay destacar que este proceso migratorio es algo sustancialmente vivo. Los datos y conclusiones que arrojan este trabajo se modifican día a día. Cualquier estudio que se haga sobre este fenómeno de la inmigración queda obsoleto en el mismo momento de su finalización. La llegada de nuevos vecinos a cualquier población altera de manera significativa la vida de ésta, siendo por tanto el problema de convivencia e integración de estas personas, en muchos de los casos con mentalidades y formas de vida totalmente diferentes a las nuestras, uno de los mayores retos que plantea en este momento la inmigración masiva a España. El análisis de estos problemas y las soluciones a estos retos están fuera del alcance de este estudio, pero quedan anotados para superar la fría estadística que acompaña a este estudio, demasiado fría cuando se trata de analizar un fenómeno protagonizado por seres humanos, los cuales en ningún momento deben de ser considerados como simples cifras, sino que debemos tener siempre presente que detrás de cada número hay una persona que ha puesto su esperanza de mejora de vida en nuestras manos y en nuestro país. Obligación moral nuestra es por tanto facilitar la integración en nuestra sociedad a estos semejantes nuestros.

\section{FUENTES Y BIBLIOGRAFIA EMPLEADAS PARA LA ELABORACIÓN DEL TRABAJO:}

- El impacto de la inmigración en una sociedad que se transforma. Miguel Laparra y otros. Gobierno de Navarra.2005.

- Instituto Nacional de Estadística.

- Instituto de Estadística de Navarra.

- Ayuntamiento de Fustiñana.

- Sindicato agrario EHNE ( COAG)

- Servicio Navarro de Empleo.

Así mismo se ha realizado un trabajo de campo para verificar dónde trabajan los inmigrantes en Fustiñana y dónde residen. Para ello se han recorrido las empresas que los contratan, entrevistando a sus responsables. También se ha recorrido el municipio comprobando las viviendas que residen los inmigrantes y verificando, a través de entrevistas, su régimen de tenencia. 